\title{
Control of proton concentration in water and organic solvents using electrochemically generated acid in very small footprint electrochemi- cal cells.
}

\author{
Janwa El-MAISS, Divya Balakrishnan and César Pascual García*
}

Nanomaterials Unit of the Materials Research and Technology Department, Luxembourg Institute of Science and Technology (LIST), Belvaux L-4422, Luxembourg.

KEYWORDS: pH control, Carboxyfluorescein, electrochemical generation of acid, microreactor, Acid-labile group deprotection

\begin{abstract}
In this work, we show the control of acidity and chemical reactions in nanofluidic electrochemical reactors with water and organic solvents. We demonstrate the accurate control of the proton concentration using Faradaic currents calibrated against carboxyfluorescein adjusted with external titrations, but also with a tautomer transition occurring at $\mathrm{pH} 4.2$. We deployed our platform for the control of acidity with organic solvents using a modification of the electrodes with a sulfonated tetrafluoroethylene-based membrane, that isolates the acid generating electrodes from the reagents in the solution. This configuration allowed us to follow the acidity in the cell using the same carboxyfluorescein, observing no deterioration of the acid/base cycles, proving the effective isolation of the electrodes. Finally, due to the high proton concentration and the acidity contrast between the cell and the outside that can be achieved with our system, we performed the deprotection of acid labile groups in an upstanding glass in a region with $150 \mu \mathrm{m}$ of diameter. To the best of our knowledge, this platform shows the best control of acidity in the smallest volume reported so far.
\end{abstract}

\section{INTRODUCTION}

Acidity has a defining role in numerous chemical and biological processes ${ }^{1}$, for example, the activity of enzymes ${ }^{2}$, the assembly of DNA ${ }^{3}$ and solid phase synthesis of biopolymers using acid or base labile protecting groups ${ }^{4,5}$. For all these applications, it is important to control and have a way to predict the acidity, which in most of the cases is done by titrating solutions of compounds dissociated in water or organic solvents. The activity of these compounds in water is predicted with the proton dissociation constant (pKa expressed in logarithm scale), which is well known for most chemicals. However, in organic solutions, the proton activity is more elusive to determine because the $\mathrm{pK}_{\mathrm{a}}$ of the conjugated acid/base pairs depends also on their interaction with the solvents, and the possible proton carriers that may be added to facilitate the dissociation, or as in the case of electrolytes, to change the properties of the solution ${ }^{6}$. Such is the case, that most of the protocols describing organic solutions provide the concentration of the acid rather than the proton activity.

To predict the acidity in organic solvents, the Bordwell $\mathrm{pK}_{\mathrm{a}}$ tables compile the information of the proton dissociation constants of around 2000 acids measured in DMSO. These tables were created mainly by the spectrophotometric titration of the acids to determine its acidity relative to some indicator acid, whose conjugate anion was colored. ${ }^{7-15}$ After Bordwell's work, other contributions completed the information with data relative to $\mathrm{pK}_{\mathrm{a}}$ values in other solvents obtained from different experiments including UV-vis measurements $^{16,17}$, potentiometry ${ }^{18-20}$, quantum chemistry ${ }^{21-23}$, magnetic nuclear resonance ${ }^{24}$ and other indirect measurements of the relative acidity and basicity of the compounds. For practical reasons, potentiometric acidity sensors like glass electrodes or ISFETs are the most used $\mathrm{pH}$ meters in water ${ }^{25-28}$. However, in organic media, they exhibit a stronger drift and often deviate from a linear behaviour ${ }^{29,30}$. When the absolute dissociation constant is not known, a relative dissociation constant can also be derived comparing the relative $\mathrm{pK}_{\mathrm{a}}$ of a substance to a reference acid ${ }^{6,31,32}$. However, the task to complete these tables flops because the counter-ions of the reference acid may have interactions with the substance to which the $\mathrm{pK}_{\mathrm{a}}$ is unknown, and often 

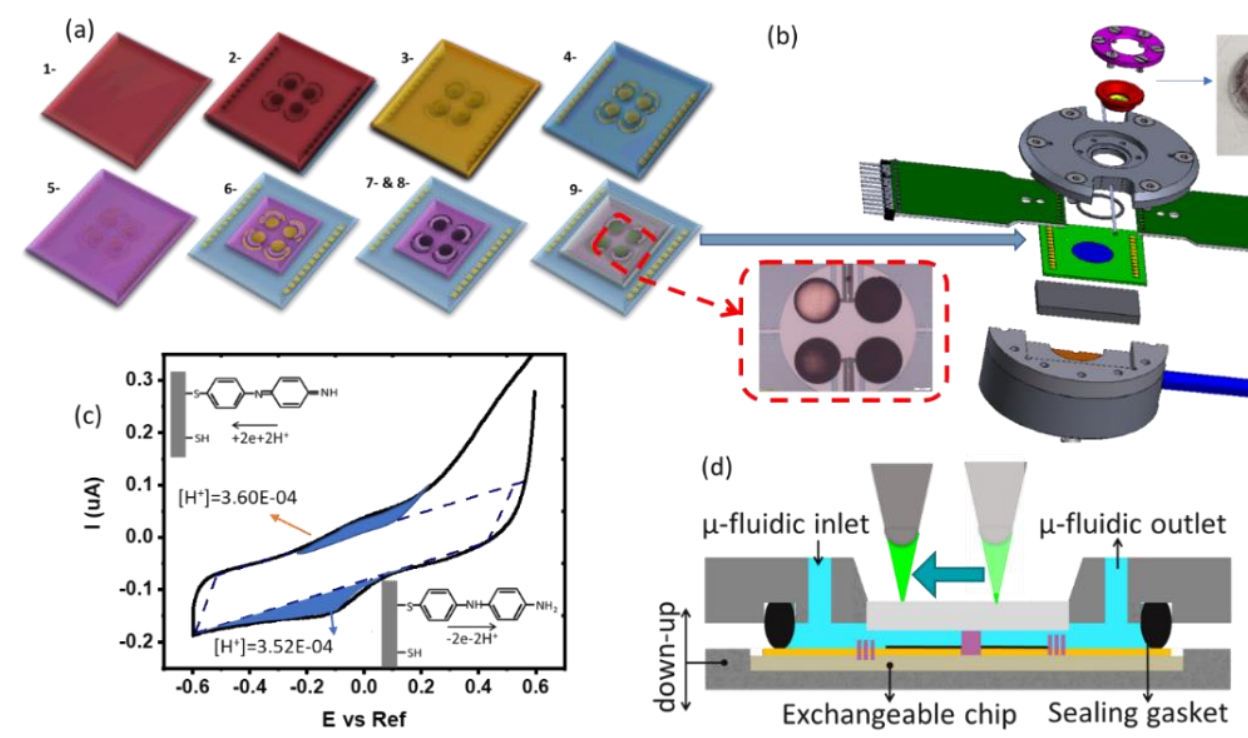

Figure 1: (a) Chip fabrication of the integrated electrochemical cells. (b) Microscope image of a device chip containing four fully functional electrochemical microreactors, and the exploded view of the platform (c) Cyclic voltammogram with the extracted Faradic currents represented with blue shadows. (d) Schematic representation of the microfluidic platform with movable optical setup on top.

additives included in the solution can radically change the behaviour of the acids.

To control the proton concentration in different solvents, our strategy was to use electrochemically generated acid that was monitored by a reference fluorescence label. We used a design of an electrochemical reactor previously proposed by us, where the acidity is generated by proton exchange reactions ${ }^{33}$. The platform holds several miniaturized electrochemical reactors each of these consists of three electrochemical partial cells containing the working, counter, and reference electrodes (WE, CE and RE, respectively) and separated by diffusion barriers. In the cell containing the WE, the acidity is regulated by a voltage bias applied respect to the reference cell, which controls the release or adsorption of protons by molecules functionalized in the WE. The electrodes are isolated from the electrolyte using a fluorinated proton permeable membrane (Nafion) ${ }^{34}$. The diffusion barriers assure that the number of protons in each cell is stable for periods much longer than the dissociation dynamics, while the small dimensions of the cell assure a rapid feedback of the reactions in the cell. The electrochemically generated acid released protons, free of counter ions and free to interact with the elements of the electrolyte. Thanks to these characteristics, the acidity can be controlled and estimated by the faradaic currents.

To monitor the proton activity in our cell, we used the fluorescent dye carboxyfluorescein (FAM). FAM allowed a calibration using an external titration that coincided with the proton concentration calculated using the Faradaic currents. We also calibrated the system using a special tautomerisation process that FAM undergoes at a particular $\mathrm{pH}$, and we used the proton quantification to control the acidity in acetonitrile (ACN). We used the fluorescence to calibrate the proton activity of acids in an organic electrolyte, observing the formation of super-acids due to the influence of proton carrier salts in the electrolyte. Finally, we used our electrochemical cell for the deprotection of acid labile groups. In our case we proved the deprotection of ter-
Buthyloxycarbonyl, (Boc) groups from an APTES monolayer grafted on a glass surface upstanding to the electrodes in the cell of the WE. This kind of reactions added a proof of the activity of electrochemically generated acid, which in traditional chemistry is obtained after using high acid concentrations.

\section{MATERIALS AND METHODS}

\section{CHEMICALS AND INSTRUMENTATION}

4-Aminothiolphenol (4ATP), Potassium chloride (KCl), 6Carboxyfluorescein(FAM), Tetrabutylammonium hexafluorophosphate $\left(\mathrm{Bu}_{4} \mathrm{PF}_{6}\right)$, Hydrochloric acid $(\mathrm{HCl})$, Potassium Hydroxide (KOH), Benzotriazol-1-yloxytripyrrolidinophosphonium hexafluorophosphate (Pybop), N,N-Diisopropylethylamine (DIPEA), 3-(Aminopropyl)triethoxysilane (APTES), Trifluoroacetic acid (TFA), Rhodamine B isothiocyanate (RITC), absolute ethanol (EtOH), anhydrous Acetonitrile (ACN), anhydrous Dichloromethane (DCM) and anhydrous Dimethyl sulfoxide (DMSO) were purchased from Sigma Aldrich and N-tert-Butyloxycarbonyl-12-amino-dodecanoic acid (acid-Boc) was purchased from Iris biotech GMBH. All the chemicals were used without further purification. For the preparation of aqueous electrolyte solutions and cleaning purposes Millipore filtered water was used. For the electropolymerization and $\mathrm{pH}$ control experiments a Solatron Modulab XM Pstat $1 \mathrm{mS} / \mathrm{s}$ potentiostat (Pstat) was used.

\section{FABRICATION OF MICROREACTORS AND THE MICROFLUIDIC PLATFORM}

The electrochemical microreactors were fabricated on Silicon chips coated with a dielectric layer of $\mathrm{SiO}_{2}$ using standard techniques of micro-electromechanical systems (MEMS) and chemical and electrochemical surface functionalization methods (fig.1(a)). The chips were integrated with a platform (fig.1(b)) having microfluidic, optical, and electrical accesses and a pneumatic actuation to open and close 
the cells. The first steps of our chip fabrication are equivalent to the ones detailed in our previous publication ${ }^{35}$ (all the details involved in the fabrication process are also reported in the supporting information (SI-1)). Briefly, optical lithography was performed to pattern the design of the electrodes. The substrate was spin-coated with an optical resist and the electrodes with contact pads were exposed and developed. Later, 5 and $50 \mathrm{~nm}$ layers of $\mathrm{Cr}$ and Au were evaporated using an e-beam evaporator. The chip was placed in acetone for lift-off. We then spin coated the chip with SU8 3010 epoxy resist to lithograph the separation barriers. The chip was afterwards developed and hard baked for 60 minutes. The electrodes were electrochemically platinized to increase their surface area ${ }^{36}$. The chip was later activated in a UV ozone cleaner for 30 minutes and functionalized with $0.5 \mathrm{mM}$ of 4-Aminothiolphenol (4ATP) in absolute ethanol for 24 hours to form a self-assembled monolayer of the redox active molecules on top of the porous electrodes. This configuration without further modifications was used for experiments with aqueous solutions. The essential difference that we used for the experiments with organic solvents is the isolation of the electrodes from the electrolyte using a sulfonated tetrafluoroethylene (Nafion) based membrane. We incorporated Nafion film by spin-coating its commercial solution after the functionalization of chip with the 4ATP molecules. The Nafion membrane, while avoiding the possibility of cross reactions between 4ATP molecules and the carboxyfluorescein, it allows the selective transport of protons from the electrodes to the solution through its sulfonate channels.

The platform consists of a central liquid reservoir delimited by a circular FFKM gasket. The configuration of the top element allows to control the aperture of the cell between an average of $100 \mu \mathrm{m}$ in the open position and a closed position, where the barriers delimiting the cells are in contact with the upstanding glass, and the gap in the electrochemical cells of the WE, CE and RE and the diffusion barriers have an average height of $\sim 5 \mu \mathrm{m}$. The open and close position were actioned by a pneumatic piston. When the cell is in the close position the total volume of the isolated partial cell of the WE $(V)$ is $0.9 \mathrm{nl}$. This volume is considered in the article to calculate the concentrations of electrochemically generated acid confined in the volume of the partial cell of the WE. The liquid in the central aperture was managed by syringe pumps through an inlet and evacuated by an outlet in which vacuum could also be applied to achieve a good filling. Finally, this cell also allowed an optical access into the central aperture through the upstanding glass (this glass will be functionalized in Boc-deprotection experiments). Using an optical microscope, we could follow the fluorescence in the electrolyte, being able to resolve the acidity sequentially between the cells.

\section{PREPARATION FOR THE FLUORESCENCE BUFFERS}

The buffers solutions employed to solubilize FAM $(0.5 \mu \mathrm{M})$ during the experiments were (a) $\mathrm{KCl}(100 \mathrm{mM})$ with a $\mathrm{pH}$ adjusted at 7 using $\mathrm{KOH}$ solution for the aqueous experiments with no tautomerization and (b) $\mathrm{KCl}(100 \mathrm{mM})$ with no $\mathrm{pH}$ adjustment for tautomeric identification. These solutions were prepared using a MilliQ water (resistivity of 18.2 $\mathrm{M} \Omega . \mathrm{cm}$ at $25^{\circ} \mathrm{C}$ ).

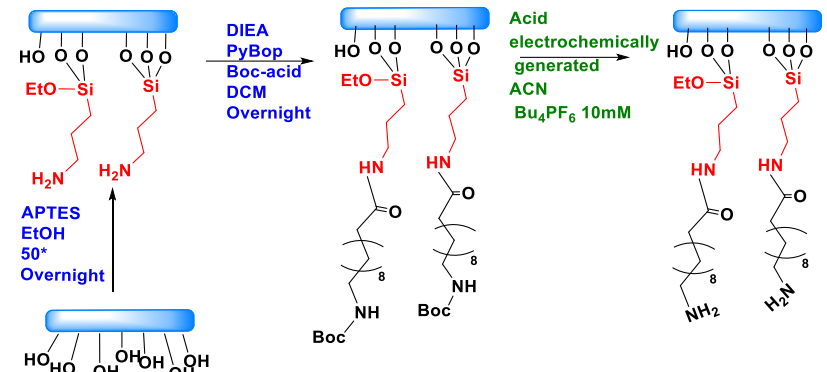

Figure 2: Functionnalization of the glass: (a) APTES 5\% v/v in absolute EtOH at $50^{\circ} \mathrm{C}$ overnight (b) DIEA 20eq,PyBOP 20eq,Boc-acid 20eq in DCM overnight (c) Electrochemical deprotection of Boc in $A C N 10 \mathrm{mM} \mathrm{Bu}_{4} \mathrm{PF}_{6}$

For the experiments in organic solvents, all the solutions used for $\mathrm{pH}$ monitoring, as well as acid titrations were prepared with the same ionic strength $10 \mathrm{mM}$ of $\mathrm{Bu}_{4} \mathrm{PF}_{6}$ (FAM $0.5 \mathrm{Mm}$ ) in ACN.

\section{FLUORESCENCE MEASUREMENTS}

Carboxyfluorescein (FAM) was used to monitor the $\mathrm{pH}$ changes inside the electrochemical cells. The fluorescence set-up was made with an Olympus BX customised microscope, using a led source centred at $470 \mathrm{~nm}$ (CoolLEDpE40000). Light was directed through a collimator and through a band pass filter, $460-500 \mathrm{~nm}$ mounted with a dichroic mirror CROMA-49012 that has also a low pass filter at $510 \mathrm{~nm}$ for collection. The excited area was limited by a diaphragm limiting the field of view to $\sim 50 \mu \mathrm{m}$. The light was collected by a concave mirror, focusing the light from the field of view at the entrance of an optic fibre that sent the signal to a wide range spectrometer MAYA-S-VIS-NIR. For the real time measurements, we followed the detection of FAM at obtaining the peak signal at $525 \mathrm{~nm}$ using an integration time of $1 \mathrm{~s}$. The schematic representation of the setup is available in SI-1

\section{BOC FUNCTIONALIZATION OF GLASSES}

For the experiments showing the deprotection of acid labile groups we functionalised circular microscope slides of $1 \mathrm{~cm}$ diameter. The glass slides were cleaned using isopropanol and acetone and blow dried with nitrogen gas. They were activated using an UV Ozone treatment for $30 \mathrm{~min}$ and immersed in a solution of $5 \%$ APTES in $40 \mathrm{ml}$ of absolute ethanol at 50 degrees overnight. The samples were cleaned and placed in a solution of PyBOP 742mg, BOC - 150mg, DIEA248ul, DCM 7.5ml (preactivated for 10mins). Samples were kept in the above solution overnight at room temperature, closed with aluminium foil. The growth of the film was followed by water contact angle and XPS (details in the SI.2). For each experiment, a functionalized glass was mounted in the upstanding lid of the platform, using a dedicated glue. Following the experiments, the glasses were removed from the platform and stained with Rhodamine B isothiocyanate to observe the deprotection fingerprint.

Two control samples were prepared for the evaluation of Boc deprotection. A negative control was used with the Boc protected samples and incubated into the Rhodamine without further treatment. The positive control was prepared after a treatment with 50\% TFA acid for one hour, before the 


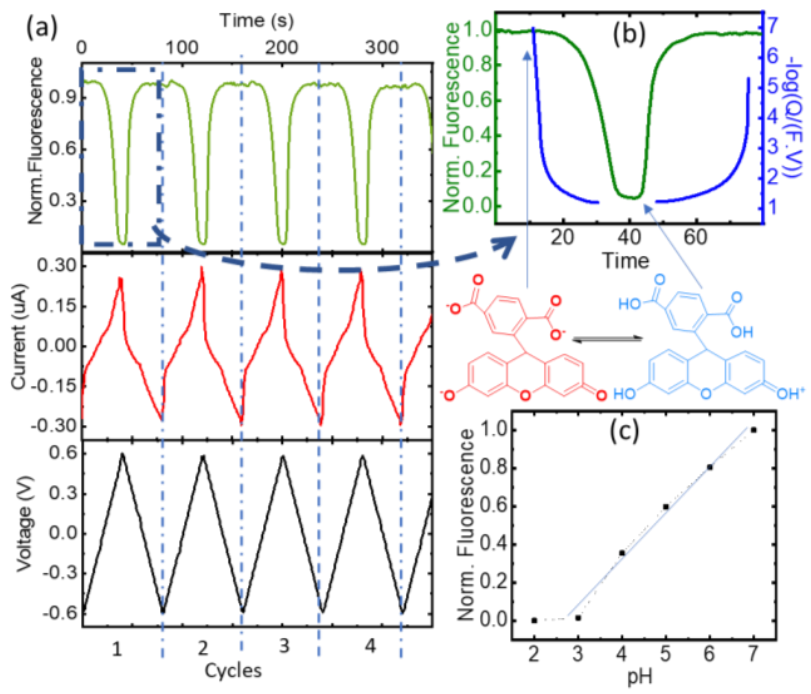

Figure 3: (a) the time evolution of the $C V$ and fluorescence data corresponding cycles 2 to 5 , the voltage, the current, and the normalized FAM-fluorescence in black, red, and green, respectively (b) Normalized fluorescence (green) and Faradaic charge expressed as apparent pH $\left(-\log Q /\left(F^{*} V\right)\right.$ ) (in blue) vs time. The different active species are indicated. (c) Calibration curve of carboxyfluorescein vs pH using external titration

same incubation with Rhodamine. The fluorescence was evaluated normalizing a spot with a field of view $\sim 50 \mu \mathrm{m}$ to the signal from an equivalent glass treated with traditional deprotection in TFA acid.

\section{CONTROL OF REDOX REACTIONS IN THE PLATFORM}

In our platform, the 4ATP functionalised electrodes can be biased to exchange protons with the electrolyte. This reaction is more efficient after the dimerization of $4 \mathrm{ATP}^{37}$. The dimerization was carried out in water by electro-polymerisation with cyclic voltammetry. Before cycling the acid/base control, the cell was placed in the open position and the electrodes were connected to the potentiostat using a three-electrodes system. A cyclic voltammetry (CV) program was applied in the range of -0.6 to $+0.6 \mathrm{~V}$ at a scan rate of $25 \mathrm{mV} / \mathrm{s}$ for 5 cycles in presence of the dye (FAM $0.5 \mathrm{uM}$ ) in $\mathrm{KCl}(100 \mathrm{mM})$ (fig.1(c)). The voltammograms corresponding to the electropolymerisation exhibited two main contributions in the oxidation peaks corresponding to the reversible exchange of protons and the dimerization at $\sim 0.1$ and $0.4 \mathrm{~V}$ respectively. As consecutive cycles were performed the main peak corresponding to the dimerization disappeared and the main contribution remained by the peak at $0.2 \mathrm{~V}$ corresponding to the two proton exchange reactions. The details of all these reactions were studied in our previous article ${ }^{37}$. In some of the chips, other residual contributions that we ascribed to non-dimerised molecules could also be observed between the two peaks of the dimerised molecules and the electropolymerisation. For the experiments in organic solvents, the FAM was used with the same initial concentration $0.5 \mu \mathrm{M}$ in a solution of $\mathrm{Bu}_{4} \mathrm{PF}_{6} 100 \mathrm{mM}$ in ACN. In organic medium, the CVs were performed between 0 to $1 \mathrm{~V}$ vs pseudo reference with a scan rate of $30 \mathrm{mV} / \mathrm{s}$. The deprotection of Boc acid labile groups occurs with the application of a linear swipe up from 0 to $0.85 \mathrm{~V}$, a fixed voltage $(0.85 \mathrm{~V})$ for $1 \mathrm{~min}$ and then a second linear swipe up from 0.85 to $0 \mathrm{~V}$.

\section{RESULTS AND DISCUSSIONS}

After the polymerization, 4-ATP dimerizes to a redox molecular state that at low applied voltages exchanges two protons with the electrolyte for each dimer in the electrode (quasi)-reversibly, and each proton is compensated by the current of one electron exchanged in the $\mathrm{WE}^{37-39}$. The CE had the same composition; thus, it balanced the reactions in the WE by the reverse proton exchange reactions. However, since the separation by the diffusive barriers keeps both partial cells apart, they do not affect each other. The Faradaic currents were fitted considering the contribution of the reversible proton exchange peaks after subtraction of the contributions from capacitance, the linear currents attributed to mass transport and the from the non-reversible residual polymerisation. The resulting Faradaic contributions of the proton exchange reactions were used to calculate the proton concentration generated at the oxidation and re-adsorbed by the electrodes during the reduction cycle (details of the calculations shown in SI-3). The calculations showed similar concentration in the two cases (in the case of fig.1(c), for instance, 3,6.10-4 $\mathrm{M}$ and 3,52.10-4 $\mathrm{M}$ were found for the oxidation and reduction peaks, respectively). This behaviour was observed for repetitive cycles (up to hundreds of cycles that we monitored the currents in some of our experiments) validating the quasi-reversibility of the system. During different experiments using different chips we observed a variety of surface functionalization in the electrodes that resulted in different dynamic ranges of $\mathrm{pH}$ obtained during the CV cycles, with typical maximum acidities reaching $\mathrm{pH}$ between 3 and 1 .

\section{MONITORING ACIDITY WITH FAM IN AQUEOUS SOLUTIONS}

The changes in FAM fluorescence $(\mathrm{pH} 7$ fixed, $0.5 \mu \mathrm{M}$ solution in $\mathrm{KCl} 100 \mathrm{mM}$ ) with the applied voltage were tracked focusing the microscope in the WE during the CV measurements and following the peak emission at $525 \mathrm{~nm}$. Figure 3 (a) reports the time evolution of the $\mathrm{CV}$ and fluorescence data corresponding to 4 representative cycles (from cycle 2 to 5, excluding the first oxidation/reduction that usually exhibit spurious contributions from the open circuit state) showing the voltage, the current, and the normalized FAMfluorescence in black, red, and green, respectively. The current curves reported the typical redox peaks corresponding to the proton exchange reactions, from which for each cycle the faradaic currents could be extracted (quantitative data of the cycles and details of the calculations shown in SI-4). We attribute the decrease of FAM fluorescence to the increase of acidity in the cell due to the proton exchange reactions. Figure 3(b) shows the details in one cycle with the FAM fluorescence (in green) and the calculated charge exchanged, $Q$ (blue curve) as function of time extracted from the Faradaic currents. $Q(t)$ is expressed as the negative logarithm scale normalised to the Faraday number and the volume of the cell, to which we added a baseline of $Q(0)=10^{7}$ (in units of elementary charge) where the exponent 7 corresponds to a baseline of neutral $\mathrm{pH}$ to account for the initial conditions of the electrolyte. In this way the charge is 

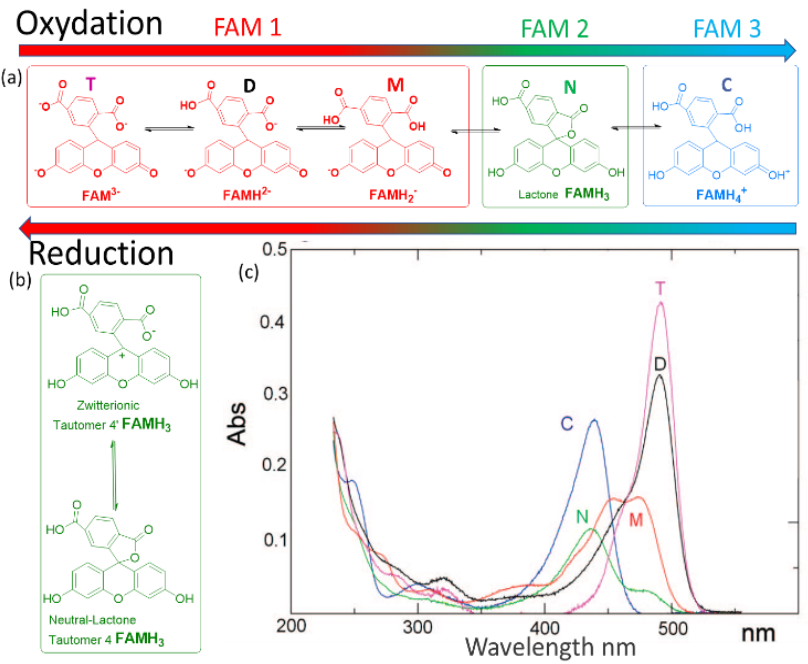

Figure 4: FAM acid/base pairs $\left(\mathrm{FAMH}_{4}^{+}\right.$cation $\mathrm{C}, \mathrm{FAMH}_{3}$ neutral $\mathrm{N}, \mathrm{FAMH}_{2}^{-}$monoanion $\mathrm{M}, \mathrm{FAMH} \mathrm{H}^{2-}$ dianion $\mathrm{D}$, and $F A M^{3-}$ trianion T) present during the oxidation and reduction process. FAM 1 is the red shifted, FAM 2 green and FAM3bleu shifted species (b) Tautomerization between the lactone FAMH3 and the zwitterionic tautomer, (c) absorption spectrum of the different species of FAM reported with permission from literature

expressed in equivalent units of pH. Figure 3(c) shows the fluorescence curve or the FAM vs. $\mathrm{pH}$, calibrated with an ISFET $\mathrm{pH}$ meter in a solution that was titrated with KOH. We observed a small delay of the fluorescence respect to the Faradaic currents part of which $(\sim 1 \mathrm{~s})$ can be attributed to the integration time of our spectra, but we did not consider this delay relevant in the overall cycle. We also observed a very good correspondence of the fluorescence obtained in the cell at the equivalent $\mathrm{pH}$ calculated by $Q$ and the one at $\mathrm{pH}$ produced with the external titration. The maximum fluorescence is obtained when no protons were released in the system or when they are reabsorbed by the 4ATP molecules after the reduction cycles that corresponds to the moment when the normalised Faradaic currents indicate $\mathrm{pH}$ 7. The intensity decreases when the oxidation of the 4-ATP occurs $(-0.6$ to $0.6 \mathrm{~V})$ and then increases during the reduction fragment of the cycle $(0.6$ to $-0.6 \mathrm{~V})$ where the protons were readsorbed. This returning to the normal fluorescence before the protons activity is also consistent with our interpretation that the phenomenon is quasi-reversible, and that the adsorption of the protons released in the system is efficient. In the cycle we detail here, the equivalent $\mathrm{pH}$ of the Faradic currents reached values close to 1 .

An independent calibration of the relation of the $Q$ derived from the Faradaic currents with the acidity was observed with the Tautomerization of FAM. Tautomerism is a solvent-dependent chemical process that commonly results in the relocation of a hydrogen atom and affects the activity of biomolecules and dyes. Either an acid or a base can catalyse the proton transfer ${ }^{40}$. FAM has different forms (each one having different tautomers that can coexist in water), and the transition from one to another can be driven by acidity. In aqueous solution, FAM has different acid/base pairs (cation $\mathrm{C}$, neutral $\mathrm{N}$, monoanion $\mathrm{M}$, dianion $\mathrm{D}$, and trianion $\mathrm{T}$ ) (a)

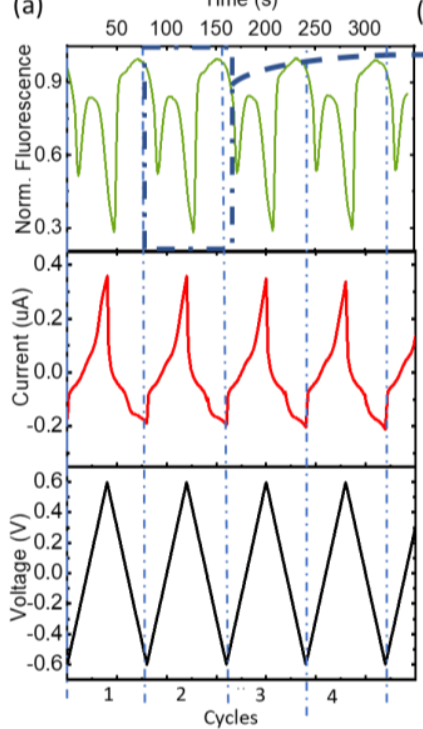

(b)
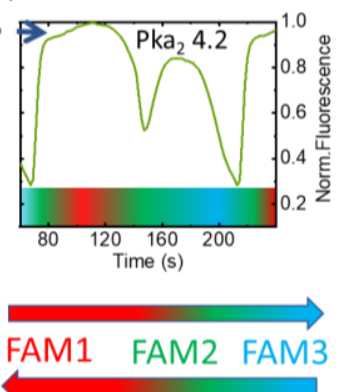

Figure 5: (a) the time evolution of the $C V$ and fluorescence data corresponding to 4 representative cycles, the voltage, the current, and the normalized FAM-fluorescence in black, red, and green, respectively, (b) Normalized fluorescence vs time and the different species in the medium 1 TO FAM3. (c) proton concentration $\left(-\log Q /\left(F^{*} V\right)\right)$ vs time and the different species in the medium

depending on the $\mathrm{pH}$. Aschi et al. ${ }^{40}$ were the first to present accurate measures of the $\mathrm{pKa}$ values of the different pairs of FAM using the spectrophotometric titration, the chemometric analysis as well as quantum mechanical estimations. Based on their paper, we adapted first the nomenclature of the different species by considering the global charge of the tautomer with the groups: i) the anion family ( $\mathrm{T}\left(\mathrm{FAM}^{3-}\right)$, D $\left(\mathrm{FAMH}^{2-}\right)$ and $\mathrm{M}\left(\mathrm{FAMH}_{2}^{-}\right)$) that had a similar absorbance at $470 \mathrm{~nm}$ (though $\mathrm{T}$, and $\mathrm{D}$ had their maximum at lower frequencies) designated as FAM1, ii) the neutral tautomer $\mathrm{N}$ $\left(\mathrm{FAMH}_{3}\right)$, which had the lowest absorbance at $470 \mathrm{~nm}$ designated as FAM2 and iii) the cation compound $\mathrm{C}\left(\mathrm{FAMH}_{4}{ }^{+}\right)$ that increased the absorbance at $470 \mathrm{~nm}$ respect to FAM 2 but not respect FAM 3. FAM1 to FAM3 are represented in fig. 4.

To observe the tautomer transition, we used freshly purchased FAM prepared from a completely dehydroxylated state (FAM1, shown in red in fig. 4) without stabilisation of the buffer. Figure 5(a) shows the time dependent curves of the voltage (black), current (red) and the fluorescence (green) from the cell containing the WE. The interpretation of the cyclic voltammograms does not change, as there is no influence of the state of compounds on the electrolyte. All the peaks were fitted to extract $Q(t)$ from the Faradic currents. However, for this preparation of the fluorophore in the electrolyte, we observed a different behaviour. Figure 5 (b) zooms into the fluorescence of cycle 2 (extended for a time slightly before and after), which is representative of the behaviour observed. The most notorious feature is the relative minimum of the fluorescence that can be observed around $150 \mathrm{~s}$ occurring at the middle of the oxidation cycle. As the voltammogram continued to complete the oxidation, the descending fluorescence with the oxidation cycle was retrieved to achieve the global minimum at the end of the 
oxidation cycle and then the maximum of the fluorescence was recovered after the reduction (in this cycle after $210 \mathrm{~s}$ ). We interpreted the intermediate minimum with the formation of a medium tautomer FAM2, less fluorescent than the species generated at this instant in the solution with a limited lifetime (few microseconds). This sudden drop in the fluorescence can be explained based on the absorption spectra (fig. 4(c)) of the different FAM species. The starting species FAM1 (shown in red) transition from one acid/base pair to another with a similar in absorbance at our excitation wavelength $(470 \mathrm{~nm})$. However, the lower absorbance of the neutral lactone N (FAM2 show in green in fig. 4(c)) at the excitation wavelengths translates into lower emission fluorescence. At this specific moment we recorded the tautomerisation of the keto form (Lactone $\mathrm{FAMH}_{3}$ ) into the zwitterionic equivalent (one of them being less fluorescent than the other). The colored band at the bottom of the fluorescence spectra represents the $\mathrm{pH}$ dependence of the different FAM acid/base pairs present in the solution and its evolution with time. Later the transition to cationic FAM3 recovers the normal behaviour. Figure 5(c) shows the calculated charge exchanged as function of time $(\mathrm{Q}(\mathrm{t}))$ extracted from the Faradaic currents during the oxidation as detailed before. It shows that the $\mathrm{pH}$ was varying in the solution via electrochemical generated acid from 7 to 2 passing therefore through all the $\mathrm{pK}_{\mathrm{a}}$ values of the FAM. Thus, as the generation of protons during the oxidation protonates first the triaonic FAM $^{3-}$ it passes by the neutral (zwitterionic) $\mathrm{FAMH}_{3}$ leading at the end to the cationic non-fluorescent $\mathrm{FAMH}_{4}{ }^{+}$. During the reduction of the ATP, the protons are re-adsorbed leading to the reverse exchange phenomenon (fig 4(a)), but the tautomerization is no longer observed, showing the instability of the neutral Tautomer $\mathrm{N}$.

Figure 4(b) and (c) represent respectively the tautomerization of the neutral tautomer into its zwitterionic state, and the absorbance spectra of all the compounds as reported in the work of Aschi et al. ${ }^{40}$. This interpretation is consistent with their measurement for the $\mathrm{pK}_{\mathrm{a}}$ values of the different families:

$$
\begin{aligned}
& \text { at } \mathrm{pKa}_{4}=\mathrm{FAM}^{3-}+\mathrm{H}_{3} \mathrm{O}^{+} \leftrightarrows \mathrm{FAMH}^{2-}+\mathrm{H}_{2} \mathrm{O} \text { Highly Fluorescent } \\
& \text { at } \mathrm{pKa}_{3}=\mathrm{FAMH}^{2-}+\mathrm{H}_{3} \mathrm{O}^{+} \leftrightarrows \mathrm{FAMH}_{2}^{-}+\mathrm{H}_{2} \mathrm{O} \\
& \text { at } \mathrm{pKa} \mathrm{a}_{2}=\mathrm{FAMH}_{2}^{-}+\mathrm{H}_{3} \mathrm{O}^{+} \leftrightarrows \mathrm{FAMH}_{3}+\mathrm{H}_{2} \mathrm{O} \\
& \text { at } \mathrm{pKa}_{1}=\mathrm{FAMH}_{3}+\mathrm{H}_{3} \mathrm{O}^{+} \leftrightarrows \mathrm{FAMH}_{4}^{+}+\mathrm{H}_{2} \mathrm{O} \text { non Fluorescent }
\end{aligned}
$$

where the $\mathrm{pK}_{\mathrm{a}}$ represents the major species coexisting in the medium, and $\mathrm{H}_{3} \mathrm{O}^{+}$results from the protons generated electrochemically by the electrodes. At the equilibrium between two species, the $\mathrm{pK}_{\mathrm{a}}$ value is equivalent to the $\mathrm{pH}$. The value of the $\mathrm{pK}_{\mathrm{a}}$ was calculated integrating the area of the oxidation peak from the beginning of the oxidation to the moment of the tautomerisation. The $\mathrm{pH}$ for the minimum of fluorescence observed at $120 \mathrm{~s}$, calculated from the Faradaic currents was 4.2 , which is in line with the $\mathrm{pKa}$ values reported in literature.

\section{CONTROL OF ACIDITY IN ORGANIC SOLVENTS.}

Following the validation of the method to calculate the proton concentration with the Faradaic currents, we deployed the platform for the control of acidity in organic solvents using ACN. Since FAM bares 2 carboxylic radicals, to avoid reactions with the amino groups of the 4-ATP in the electrodes we introduced a coating layer with a perfluorinated sulfonate polymer (Nafion 117). Nafion in addition to protect the ATP groups, it can transport the electrochemically generated protons from the electrodes to the solution through its sulfonate channels. Figure 6 (a) shows a few representative cycles (from 2 to 5 , also excluding the first cycle to exclude the effects from the open circuit state) from a series of voltammograms performed in the microreactor, together with the fluorescence obtained from FAM $(0.5 \mu \mathrm{M})$ dissolved in an acetonitrile-based electrolyte $(10 \mathrm{mM}$ $\mathrm{Bu}_{4} \mathrm{PF}_{6}$ ) solution. The CVs vs. time are shown representing the voltage bias in black and the measured current and fluorescence in red and green, respectively. The complete experiment was also carried on for several tenths of repetitions without apparent degradation of the Faradaic currents of the fluorescence, reflecting the efficiency of Nafion to avoid side reactions between FAM and the electrodes. Figure 6(b) shows a representative cycle of the CV (cycle 2) with the classical representation of current vs bias voltage. The Faradaic currents show two peaks that we ascribe to the oxidation of the dimerised and monomer forms of ATP at $\sim 0.3 \mathrm{~V}$ and $\sim 0.55 \mathrm{~V}$, respectively. These peaks were persistent; thus, we believe that the Nafion coating avoids further polymerisation. The oxidation peaks are also shifted towards higher bias, as it is expected due to the increase of the $\mathrm{pK}_{\mathrm{a}}$ of the electrolyte. We also observe an increase of the bias for the reduction peak, consequent with this effect. The Faradaic currents were extracted from each voltammogram cycle to calculate the number of protons produced during each bias ramp (more details of the extraction shown in SI5 Table 2). The concentration of the protons produced during the oxidation $\left(1,67.10^{-2} \mathrm{M}\right)$ is almost equivalent to ones re-adsorbed for the reduction $\left(1,45 \cdot 10^{-2} \mathrm{M}\right)$. The concentration of protons $\left(-\log \left(\mathrm{Q} / F^{*} \mathrm{~V}\right)\right)$ and the relative fluorescence normalised to the maximum value in normal conditions are shown vs time in the inset of Figure 6(c).

In acetonitrile, the electrolyte plays a key role in the mobility of the electrochemically generated protons. Different equilibria are in competition in the solution: the dissociation of the electrolyte, its association with the protons, the protonation of FAM, as well as the protonation of the solvent itself ${ }^{41}$. These equilibria can be shown as follows:

$$
\begin{array}{ll}
\mathrm{Bu}_{4} P F_{6} \leftrightarrows B u_{4}^{+}+P F_{6}^{-} & E q(1) \\
H^{+}+P F_{6}^{-} \leftrightarrows H P F_{6} & E q(2) \\
H^{+}+A C N \leftrightarrows A C N H^{+} & E q(3) \\
F_{A M}{ }^{3-}+H P F_{6} \leftrightarrows F A M H^{2-}+P F_{6}^{-} & E q(4)
\end{array}
$$

Equation (1) shows the dissociation of the salt added to provide the electrolyte conductivity. Indeed, this salt acts as the main proton carrier in the electrolyte (Eq. (2)) as $P F_{6}^{-}$has a much higher proton affinity than ACN (Eq. (3)). In our system, the protons are mainly generated electrochemically from the electrodes. They are introduced in the electrolyte without a conjugated base, which remains fixed to the electrodes and isolated from the medium using Nafion. The concentrations of protons generated is similar to the $\mathrm{Bu}_{4} \mathrm{PF}_{6}$, but 100 times higher than the concentration of FAM (FAM $0.5 \mu \mathrm{M}, \mathrm{Bu}_{4} \mathrm{PF}_{6} 10 \mathrm{mM}$ vs protons electrogenerated 


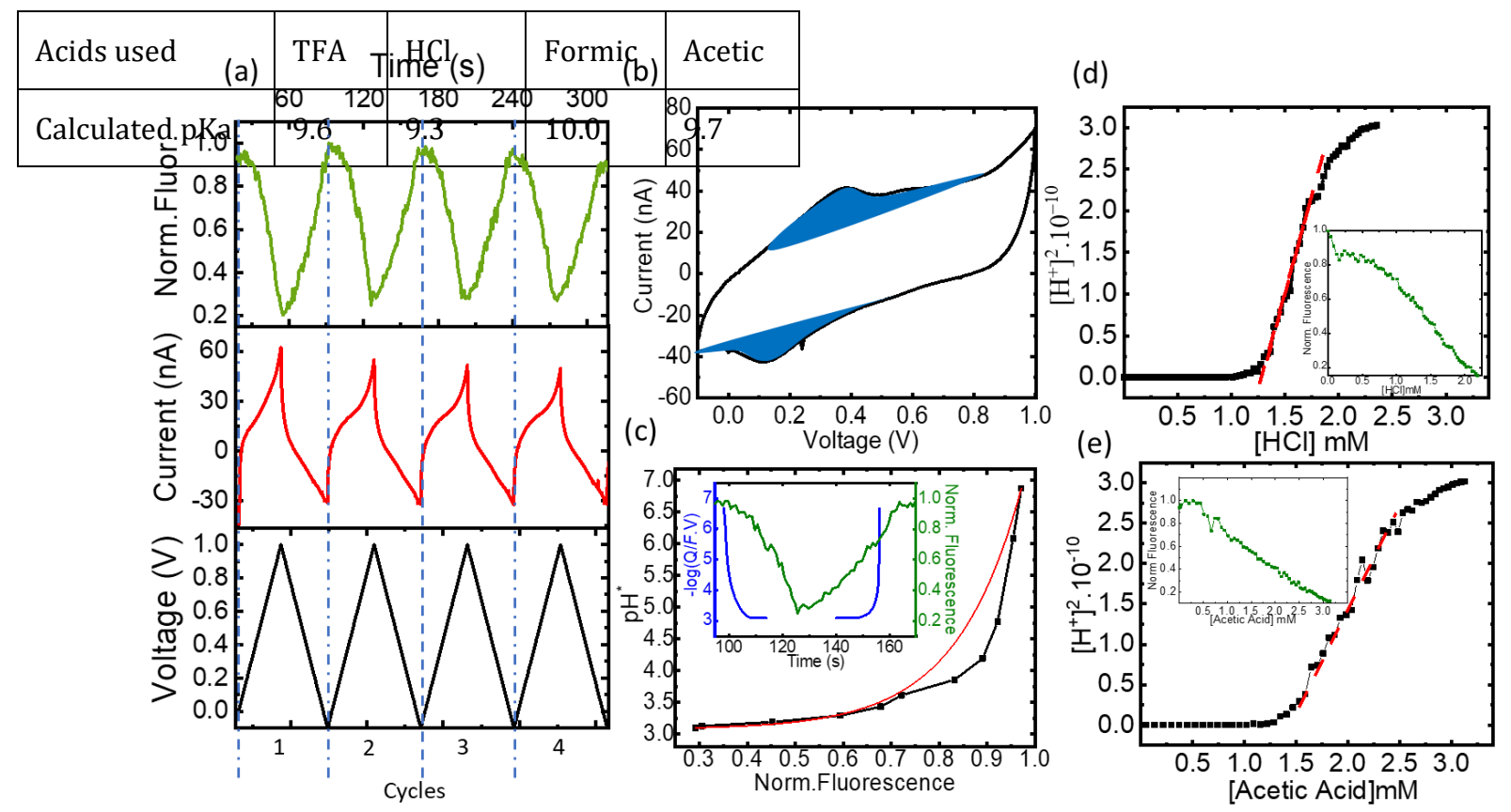

Figure 6: (a) the time evolution of the CV and fluorescence data corresponding to 4 representative cycles, the voltage, the current, and the normalized FAM-fluorescence in black, red and green, respectively, (b) Cyclic voltammogram and the extracted faradaic currents, (c) $p H^{*} v s$ Normalized fluorescence and the exponential fitting, in the inset the Normalized fluorescence (green) and proton

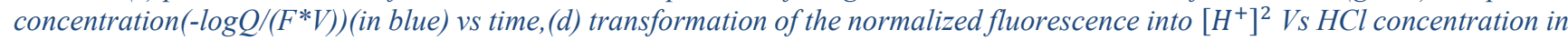
black, the slope of the corresponding curve in dashed red inset in green the titration curve of HCl, Fluorescence vs the concentration, (e) transformation of the normalized fluorescence into $\left[\mathrm{H}^{+}\right]^{2} \mathrm{Vs}$ Acetic acid concentration in black, the slope of the corresponding curve in dashed red inset in green the titration curve of Acetic acid, Fluorescence vs the concentration,

calculated from the Faradaic currents $\sim 14.5 \mathrm{mM}$ ). Thus, as the acid generated by the Faradaic currents reaches the electrolyte, the protonation of $P F_{6}^{-}$(Eq. 2) and the migration of protons to FAM (Eq. 4) (faster than the protonation of the acetonitrile (Eq. 3)) assures the availability of all the protons to interact with the acid-base pairs of the FAM. Also, due to the planar configuration of the electrochemical cell and the electrodes, the typical diffusion length is limited by the height of the cell, which is in the order of few $\mu \mathrm{m}$. Thus, the fluorescence in our electrolyte was mainly determined by the equilibrium in Eq. (4). Then we can interpret the reversibility of the fluorescence spectra from a complete quenching during the oxidation and its total recovery after the reduction bias as the reporting of FAM fluorescence of the proton activity. In the inset of fig.6 (c), we observe a delay of the fluorescence respect to the faradaic charge, which we attribute to the delay of protons to cross the Nafion membrane. However, as the time interval between the oxidation and reduction is much longer (30s), the equivalence in fig $6 \mathrm{pH}^{*}$ vs. FAM fluorescence is a good approximation over a full cycle. We can define the proton activity of electrochemically generated acid in the cell, the $\mathrm{pH}^{*}$ using the Faradaic currents $\left(-\log \left(\mathrm{Q} / F^{*} \mathrm{~V}\right)\right)$. Assuming the delay of the protons to cross the Nafion barriers, we used the correlated temporal dependence of Faradaic currents and fluorescence shown in the inset of fig. 6 (c) to generate FAM fluorescence vs. $\mathrm{pH}^{*}$ shown in black in fig. 6 .

Table 1: calculated $\mathrm{pKa}$ values for the different titrated acids
We used the relation of the $\mathrm{pH}^{*}$ with FAM fluorescence to calibrate the dissociation of acids in a solvent with the same composition of our electrolyte. We titrated different acids including strong and weak acids ( $\mathrm{HCl}$ and TFA for the strong and acetic and formic for the weak acids, respectively). We

recorded the FAM fluorescence signal corresponding to the titration of the acids using a solvent with the same composition as our medium (insets fig 6(d) and 6(e)). To obtain the $\mathrm{pK}_{\mathrm{a}}$ dependence, we considered the acid dissociation constant:

$$
K_{a}=\frac{\left[H^{+}\right] \cdot\left[A^{-}\right]}{[H A]}
$$

Since the dissociation of ACN is very low $\left(\mathrm{ACN} \mathrm{pK}_{\mathrm{a}}=40^{42}\right)$, all the protons derive from the acid, thus the proton concentration in this system can be considered the same than the conjugated base, then:

$$
K_{a}=\frac{\left[H^{+}\right]^{2}}{[H A]}
$$

and thus, we used the slope of $\left[\mathrm{H}^{+}\right]^{2}$ vs. [HA]. The proton concentration in this formula is derived from the exponential fit of the fluorescence to the $\mathrm{pH}^{*}$ shown in red in fig. 6 (c) (details of the fitting shown in SI-6), while [HA] is the concentration of the titrated acid (see Fig 7 and 8 in SI). Figures 6 (d) and (e) show the dependence for $\mathrm{HCl}$ and formic acid of $\left[\mathrm{H}^{+}\right]^{2}$ vs. $[\mathrm{HA}]$, where the linear fit is indicated in red in the central region of the dependence, outside the influence of quenching and saturation of the FAM. Table 1 reports the values found for the four acids. 

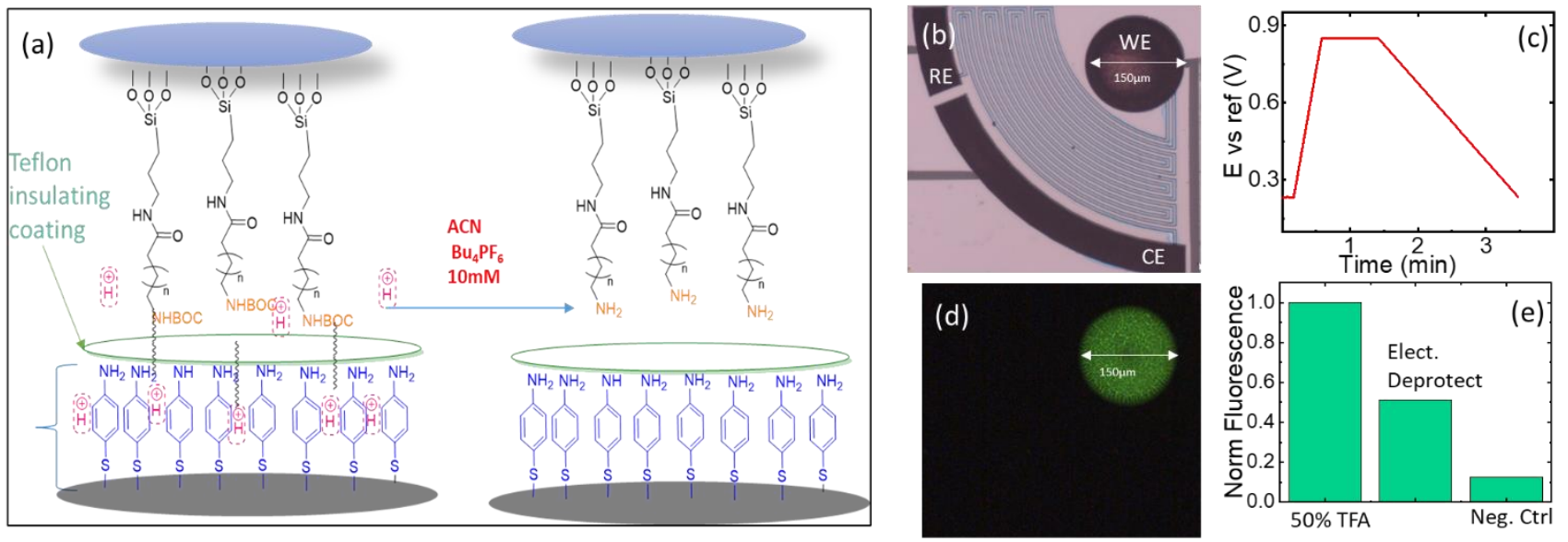

Figure 7: (a) Description of the deprotection of BOC in the electrochemical cell coated with Nafion, (b) full reactor with circular WE electrode, CE and RE in black and diffusion barriers in grey, (c) electrical bias (pulse) applied on the WE vS the RE at 0.85V for $1 \mathrm{~min}$, (d)deprotected cell after incubation in Rhodamine B, (e) comparison of the fluorescence of the electro-deprotected glass with a glass treated by the TFA $50 \%$ and negative control

It is noted that the $\mathrm{pK}_{\mathrm{a}}$ from the four acids had similar values in a range from 9 to 10 regardless some of them are considered weak acids. This similar behaviour can already be noted from the allure of the fluorescence titration curves as the fluorescence vs. acid concentration had a similar behaviour in all cases. The strong acidity observed by all the compounds is explained by the influence of the electrolyte $\mathrm{Bu}_{4} \mathrm{PF}_{6}$, that creates super-acids due to the ability of $\mathrm{PF}_{6}^{-}$to associate the protons dissociated from the acids already reported in literature ${ }^{43}$. As a result of our calibration of the acidity response of FAM with the Faradaic currents, we were able to find the activity of the acids in the complex media that included $\mathrm{Bu}_{4} \mathrm{PF}_{6}$.

\section{DEPROTECTION OF ACID LABILE GROUPS USING ELECTROCHEMICALLY GENERATED ACID.}

Following the good control of the acidity reaching very high concentrations of protons, we disposed the generation of acid to deprotect acid labile groups employed in solid phase synthesis. We tested Boc protecting group, known in peptide and nucleotide synthesis due to the high yield of polymers that it achieves. However, the high yields of purity achieved are also due to its stability that ensures the orthogonality of the chemistry during acid/base transitions and which requires a high acid concentration for its removal $(\sim 50 \%$ TFA in DCM used in many traditional solid phase synthesis).

We proceeded to use glasses functionalized with amino groups protected by a Boc terminal function as early described in fig 2. Figure 7(a) represents the deprotection of BOC groups using electrochemically generated protons by chronoamperometry from Nafion protected electrodes in the presence of the ACN based electrolyte (no FAM was included in these experiments). Figure 7(b) shows a microscope picture corresponding to one of the electrochemical cells. The black platinised electrodes are seen in the microscope in black. The electrodes are connected by diffusion barriers that are seen in fig. 7 (b) in grey. The circular electrode is the WE, while the short and long arched electrodes are the RE and CE, respectively. To be sure that we generated enough protons, a pulsed voltage bias to the WE vs. the RE was maintained at $0.85 \mathrm{~V}$ for $1 \mathrm{~min}$. Figure 7 (c) shows the electrical bias used for the generation of acid. After the exposure to this pulse, the glass was removed from the reactor and incubated with Rhodamine B isothiocyanate in DMF for an hour to let the isothiocyanate function of the fluorophore react with newly Boc-deprotected amine. Figure 7 (d) shows the resulting image of the glass after the incubation. The area of the deprotected region is equivalent to the total footprint of our WE both being spots with a radius of $\sim 150$ $\mu \mathrm{m}$. The fidelity of the fluorescence image in fig. 7(d) to the footprint of the $\mathrm{WE}$, shows the efficiency of the system to confine the acidity during the chronoamperometry experiments. This also supports the approximation that we have used through the article to consider the protons confined to the partial cell of the WE.

We compared the deprotection of electrochemically generated acid with the positive and negative controls described in the section materials and methods. The results are shown in fig. 7(e). The negative control shows that the deprotection was efficient, while the comparison with the positive control yielded lower fluorescence on the glass of the electrochemically generated acid. Nevertheless, since we cannot exclude contributions from fluorescence areas larger than our field of view (due to imperfections of the diaphragm in our microscope system) we believe the positive control is an upper limit. The deprotection achieved by the electrochemically generated acid was quite significant, considering that the deprotected area had the same footprint of the WE. Since the surface area of the platinised electrodes was $\sim 10$ times the flat area of the glass, we achieved such deprotection with $\sim 10$ molar equivalents of the exposed amino groups.

\section{CONCLUSION}

In this work, we have shown a microfluidic platform bearing an accurate control of the acidity by means of electrochemically generated acid. We have compared the calibration of 
the dependence of the FAM fluorescence with $\mathrm{pH}$ using the calculation of electrochemically generated acid in our platform and the ISFET measurement of an externally titrated acid. We have found a very good correspondence of the fluorescence values of the FAM titrated with acid and the ones produced by the Faradaic currents.

We have obtained an independent calibration of the $\mathrm{pH}$ following the tautomerization of the carboxyfluorescein. This is a delicate process, difficult to reproduce, that we were able to observe in three independent experiments in our platform using non-stabilized buffers. In our system, the tautomerization expressed in the local minimum of the FAM fluorescence due to the change of light absorption at the excitation wavelength. Also, in this case, we have found a very good correspondence of the $\mathrm{pK}_{\mathrm{a}}$ value of a tautomer transition with the $\mathrm{pH}$ of the cell, calculated with the Faradaic currents at the point where we observed the local minimum of the fluorescence. These two results confirm the possibility to use the Faradaic charge normalised to the volume of the partial cell of the WE to predict the acidity. We have also demonstrated the control of the acidity in organic solvents (in ACN), using electrochemically generated acid. Organic solvents are a delicate environment for the electrodes used in our platform because of the cross reactions that occur between the amino groups and reagents in the medium yielding to their degradations. We have shown that is possible to use a Nafion coating to separate the production of electrochemically generated acid, from the chemical reaction happening in the solution. The sulfonate channels allowed the conduction of protons from the electrodes to the electrolyte. We observed quasi-reversible cycles even at high voltages as expected by the higher $\mathrm{pK}_{\mathrm{a}}$ of the electrolyte. Neither the organic environment, nor the higher biases implied deteriorated the cyclic currents or the observed fluorescence. Thus, the acid/base cycles were quasi-reversible for tenths of cycles.

One of the advantages of the electrochemically generated acid in our system is that it does not have a conjugated base that could react and interfere in the medium. In organic acidity we used the correspondence of the faradaic currents with the proton concentration in the cell to calibrate the FAM fluorescence. Thanks to this, we were able to calculate the $\mathrm{pK}_{\mathrm{a}}$ values of four different acids in the presence of $\mathrm{Bu}_{4} \mathrm{PF}_{6}$, which are different than the values reported in literature. This task is complicated to measure otherwise by other methods, due to the different interactions of the salt, the solvent, and the acids themselves.

The maximum proton concentration achieved in the cell in some experiments was $0.1 \mathrm{M}$. Such a high electrochemically generated acidic concentration proved its efficiency by deprotecting an acid labile protecting group upstanding the partial cell of the WE in only one minute.

To the best of our knowledge, this platform shows the best control of acidity in the smallest volume reported so far. This volume is three orders of magnitude smaller than the last version that we used in our previous publication, as the footprint has decreased to the order of $150 \mu \mathrm{m}$. The high fidelity, achieved using chemical reactions to deprotect acid labile groups placed in a glass upstanding the partial cell of our WE, shows the high potential of our platform to control addressable chemical reactions. Owing the high level of miniaturization, it would be possible to highly parallelize such chemical reactions, for example, to achieve high density polymer microarrays using parallelized solid phase synthesis.

\section{ASSOCIATED CONTENT}

In the supplementary information we show in detail the fabrication steps of the electrochemical chip and the characterization of the Boc-functionalized glasses. The details about the extraction of the faradaic currents, the calculations of the $\mathrm{pH}$ and the protons concentrations in aqueous and organic solvents are shown also in SI,

\section{AUTHOR INFORMATION}

Janwa El-MAISS contributed on the design of experiments, analysis of data, writing of the manuscript and preparation of the figures. Divya Balakrishnan contributed to the design and the fabrication of electrochemical chips through optical lithography and interpretation of the experiments and César Pascual-García contributed to the design and interpretation of the experimental data and to the writing of the manuscript. The authors declare that they have no competing interests.

Corresponding author: cesar.pascual@list.lu, other authors janwa.elmaiss@list.lu,divya.balakrishnan@list.lu.

\section{ACKNOWLEDGMENT}

This project was financed by the FET-OPEN program of the European Innovation Council, Grant Number 862539.

\section{ABBREVIATIONS}

The main abbreviations used in the txt are: WE: working electrode, CE: counter electrode, RE: reference electrode, FAM: 6-carboxyfluoresceine, 4ATP: 4-Aminothiolphenol.

\section{REFERENCES}

Ghoneim, M. T.; Nguyen, A.; Dereje, N.; Huang, J.; Moore, G. C.; Murzynowski, P. J.; Dagdeviren, C. Recent Progress in Electrochemical PH-Sensing Materials and Configurations for Biomedical Applications. Chem. Rev. 2019, 119 (8), 52485297.

(2) Herlet, J.; Kornberger, P.; Roessler, B.; Glanz, J.; Schwarz, W. H.; Liebl, W.; Zverlov, V. V. A New Method to Evaluate Temperature vs. PH Activity Profiles for Biotechnological Relevant Enzymes. Biotechnol. Biofuels 2017, 10 (1), 234246.

(3) Liu, L.; Liu, J. W.; Huang, Z. M.; Wu, H.; Li, N.; Tang, L. J.; Jiang, J. H. Proton-Fueled, Reversible DNA Hybridization Chain Assembly for PH Sensing and Imaging. Anal. Chem. 2017, 89 (13), 6944-6947.

Praveen, K.; Das, S.; Dhaware, V.; Pandey, B.; Mondal, B.; Gupta, S. Sen. PH-Responsive "Supra-Amphiphilic" Nanoparticles Based on Homoarginine Polypeptides. ACS 
Appl. Bio Mater. 2019, 2 (10), 4162-4172.

Michigami, K.; Sakaguchi, T.; Takemoto, Y. Catalytic Dehydrative Peptide Synthesis with Gem-Diboronic Acids. ACS Catal. 2020, 10 (1), 683-688.

Kütt, A.; Selberg, S.; Kaljurand, I.; Tshepelevitsh, S.; Heering, A.; Darnell, A.; Kaupmees, K.; Piirsalu, M.; Leito, I. PKa Values in Organic Chemistry - Making Maximum Use of the Available Data. Tetrahedron Lett. 2018, 59 (42), 3738-3748.

Bordwell, F. G.; Liu, W. Z. Effects of Sulfenyl, Sulfinyl and Sulfonyl Groups on Acidities and Homolytic Bond Dissociation Energies of Adjacent C-H and N-H Bonds. J. Phys. Org. Chem. 1998, 11 (6), 397-406.

(8) Bordwell, F. G.; Harrelson Jr, J. A. Acidities and Homolytic Bond Dissociation Energies of the $\mathrm{AC}-\mathrm{H}$ Bonds in Ketones in DMSO. Can. J. Chem. 1990, 68 (10), 1714-1718.

(9) Bordwell, F. G.; Algrim, D. J. Acidities of Anilines in Dimethyl Sulfoxide Solution. J. Am. Chem. Soc. 1988, 110 (9), 456-463.

(10) O’Donnell, M. J.; Bennett, W. D.; Bruder, W. A.; Jacobsen, W. N.; Knuth, K.; LeClef, B.; Polt, R. L.; Bordwell, F. G.; Mrozack, S. R.; Cripe, T. A. Acidities of Glycine Schiff Bases and Alkylation of Their Conjugate Bases. J. Am. Chem. Soc. 1988, 110 (25), 8520-8525.

(11) Zhao, Y.; Bordwell, F. G. Bond Dissociation Free Energies of the H-A Bonds in HA2- Dianions. J. Org. Chem. 1995, 60 (13), 3932-3933.

(12) Bordwell, F. G.; Fried, H. E. Acidities of the H-C Protons in Carboxylic Esters, Amides, and Nitriles. J. Org. Chem. 1981, 46 (22), 4327-4331.

(13) Bordwell, F. G.; Liu, W. Z. Solvent Effects on Homolytic Bond Dissociation Energies of Hydroxylic Acids. J. Am. Chem. Soc. 1996, 118 (44), 10819-10823.

(14) Bordwell, F. G.; McCallum, R. J.; Olmstead, W. N. Acidities and Hydrogen Bonding of Phenols in Dimethyl Sulfoxide. J. Org. Chem. 1984, 49 (8), 1424-1427.

(15) Olmstead, W. N.; Margolin, Z.; Bordwell, F. G. Acidities of Water and Simple Alcohols in Dimethyl Sulfoxide Solution. J. Org. Chem. 1980, 45 (16), 3295-3299.

(16) Tshepelevitsh, S.; Kütt, A.; Lõkov, M.; Kaljurand, I.; Saame, J.; Heering, A.; Plieger, P. G.; Vianello, R.; Leito, I. On the Basicity of Organic Bases in Different Media. European Journal of Organic Chemistry. 2019, pp 6735-6748.

(17) Zhu, Z.; Odagi, M.; Supantanapong, N.; Xu, W.; Saame, J.; Kirm, H. U.; Abboud, K. A.; Leito, I.; Seidel, D. Modular Design of Chiral Conjugate-Base-Stabilized Carboxylic Acids: Catalytic Enantioselective [4+2] Cycloadditions of Acetals. J. Am. Chem. Soc. 2020, 142 (36), 15252-15258.

(18) Kaljurand, I.; Rodima, T.; Leito, I.; Koppel, I. A.; Schwesinger, R. Self-Consistent Spectrophotometric Basicity Scale in Acetonitrile Covering the Range between Pyridine and DBU.J. Org. Chem. 2000, 65 (19), 6202-6208.

(19) Koppel, I. A. The Enormous Acidifying Effect of the Supersubstituent $=$ NSO2CF3 on the Acidity of Derivatives of Benzenesulfonamide and Toluene-p-Sulfonamide in the Gas Phase and in Dimethyl Sulfoxide. J. Chem. Soc. Perkin Trans. 2 2001, No. 2, 229-232.

(20) Suu, A.; Jalukse, L.; Liigand, J.; Kruve, A.; Himmel, D.; Krossing, I.; Rosés, M.; Leito, I. Unified PH Values of Liquid Chromatography Mobile Phases. Anal. Chem. 2015, 87 (5), 2623-2630.

(21) Leito, I.; Koppel, I. A.; Koppel, I.; Kaupmees, K.; Tshepelevitsh, S.; Saame, J. Basicity Limits of Neutral Organic Superbases.
Angew. Chemie - Int. Ed. 2015, 54 (32), 9262-9265.

Kaljurand, I.; Saame, J.; Rodima, T.; Koppel, I.; Koppel, I. A.; Kögel, J. F.; Sundermeyer, J.; Köhn, U.; Coles, M. P.; Leito, I. Experimental Basicities of Phosphazene, Guanidinophosphazene, and Proton Sponge Superbases in the Gas Phase and Solution. J. Phys. Chem. A 2016, 120 (16), 2591-2604.

(23) Zhang, M.; Sonoda, T.; Mishima, M.; Honda, T.; Leito, I.; Koppel, I. A.; Bonrath, W.; Netscher, T. Gas-Phase Acidity of Bis[(Perfluoroalkyl)Sulfonyl]Imides. Effects of the Perfluoroalkyl Group on the Acidity. J. Phys. Org. Chem. 2014, 27 (8), 676-679.

(24) Parman, E.; Toom, L.; Selberg, S.; Leito, I. Determination of PKa Values of Fluorocompounds in Water Using 19F NMR. J. Phys. Org. Chem. 2019, 32 (6), 3940-3948.

(25) Bagshaw, E. A.; Wadham, J. L.; Tranter, M.; Beaton, A. D.; Hawkings, J. R.; Lamarche-Gagnon, G.; Mowlem, M. C. Measuring PH in Low Ionic Strength Glacial Meltwaters Using Ion Selective Field Effect Transistor (ISFET) Technology. Limnol. Oceanogr. Methods 2021, 19 (3), 222-233.

(26) Rani, D.; Rollo, S.; Olthuis, W.; Krishnamoorthy, S.; García, C. P. Combining Chemical Functionalization and Finfet Geometry for Field Effect Sensors as Accessible Technology to Optimize PH Sensing. Chemosensors 2021, 9 (2), 20-32.

(27) Jamasb, S. Continuous Monitoring of PH and Blood Gases Using Ion-Sensitive and Gas-Sensitive Field Effect Transistors Operating in the Amperometric Mode in Presence of Drift. Biosensors 2019, 9 (1), 44-56.

(28) López-Huerta, F.; Woo-Garcia, R. M.; Lara-Castro, M.; EstradaLópez, J. J.; Herrera-May, A. L. An Integrated ISFET PH Microsensor on a CMOS Standard Process. J. Sens. Technol. 2013, 03 (03), 57-62.

(29) Izutsu, K.; Yamamoto, H. Applicability of $0.05 \mathrm{Mol} \mathrm{Kg-1}$ Potassium Hydrogen Phthalate as Reference Value $\mathrm{PH}$ Standard in Water-Organic Solvent Mixtures. Talanta 1998, 47 (5), 1157-1163.

(30) Izutsu, K.; Yamamoto, H. Response of an Iridium Comparison with Other Oxide. Anal. Sci. 1996, 12, 905-909.

(31) Kütt, A.; Rodima, T.; Saame, J.; Raamat, E.; Mäemets, V.; Kaljurand, I.; Koppel, I. A.; Garlyauskayte, R. Y.; Yagupolskii, Y. L.; Yagupolskii, L. M.; Bernhardt, E.; Willner, H.; Leito, I. Equilibrium Acidities of Superacids. J. Org. Chem. 2011, 76 (2), 391-395.

(32) Kütt, A.; Leito, I.; Kaljurand, I.; Sooväli, L.; Vlasov, V. M.; Yagupolskii, L. M.; Koppel, I. A. A Comprehensive SelfConsistent Spectrophotometric Acidity Scale of Neutral Brønsted Acids in Acetonitrile. J. Org. Chem. 2006, 71 (7), 2829-2838.

(33) Balakrishnan, D.; Lamblin, G.; Thomann, J. S.; Van Den Berg, A.; Olthuis, W.; Pascual-García, C. Electrochemical Control of PH in Nanoliter Volumes. Nano Lett. 2018, 18 (5), 2807-2815.

(34) Umsarika, P.; Changkhamchom, S.; Paradee, N.; Sirivat, A.; Supaphol, P.; Hormnirun, P. Proton Exchange Membrane Based on Sulfonated Poly (Aromatic Imide-Co-Aliphatic Imide) for Direct Methanol Fuel Cell. Mater. Res. 2018, 21 (1), 1-8.

(35) Balakrishnan, D.; Gerard, M.; Del Frari, D.; Girod, S.; Olthuis, W.; García, C. P. Redox Active Polymer as a PH Actuator on a Re-Sealable Microfluidic Platform. J. Mater. Sci. Eng. 2018, 07 (03), 1073-1077.

(36) Frasconi, M.; Tel-Vered, R.; Elbaz, J.; Willner, I. Electrochemically Stimulated PH Changes: A Route to Control Chemical Reactivity. J. Am. Chem. Soc. 2010, 132 (6), 2029- 
Aschi, M.; D’Archivio, A. A.; Fontana, A.; Formiglio, A. Physicochemical Properties of Fluorescent Probes: Experimental and Computational Determination of the Overlapping PKa Values of Carboxyfluorescein. J. Org. Chem. 2008, 73 (9), 3411-3417.

D.; Van Den Berg, A.; Olthuis, W.; Pascual-García, C. Influence of Polymerisation on the Reversibility of Low-Energy Proton Exchange Reactions by Para-Aminothiolphenol. Sci. Rep. 2017, 7 (1), 15401-15412.

(38) Hayes, W. A.; Shannon, C. Electrochemistry of SurfaceConfined Mixed Monolayers of 4-Aminothiophenol and Thiophenol on Au. Langmuir 1996, 12 (15), 3688-3694.

(39) Raj, C. R.; Kitamura, F.; Ohsaka, T. Electrochemical and in Situ FTIR Spectroscopic Investigation on the Electrochemical Transformation of 4-Aminothiophenol on a Gold Electrode in Neutral Solution. Langmuir 2001, 17 (23), 7378-7386.

41) Malloum, A.; Conradie, J. Global and Local Minima of Protonated Acetonitrile Clusters. New J. Chem. 2020, 44 (40), 17558-17569.

(42) Kütt, A.; Tshepelevitsh, S.; Saame, J.; Lõkov, M.; Kaljurand, I.; Selberg, S.; Leito, I. Strengths of Acids in Acetonitrile. European J. Org. Chem. 2021, 2021 (9), 1407-1419.

(43) Srivastava, A. K.; Misra, N. Hydrogenated Superhalogens Behave as Superacids. Polyhedron 2015, 102, 711-714. 